\title{
Steps in the investigation and management of low semen volume in the infertile man
}

\author{
Matthew Roberts, MD, FRCSC; ${ }^{*}$ Keith Jarvi, MD, FRCSC ${ }^{\dagger}$
}

\begin{abstract}
An adequate semen volume of ejaculate fluid is required to transport sperm into the female reproductive tract and allow for fertilization of the oocyte. Thus, seminal fluid volume is an important part of the semen analysis done to investigate male infertility. In this article, we review the anatomy and physiology of ejaculation, the various etiologies of low-volume ejaculation (artifactual, structural, functional). We then present a comprehensive algorithm for the evaluation, diagnosis and treatment of the infertile man presenting with low semen volume.
\end{abstract}

Can Urol Assoc J 2009:3(6):479-85

\section{Résumé}

Un volume suffisant de liquide séminal dans l'éjaculat est nécessaire pour transporter les spermatozoïdes dans les voies reproductrices de la femme et permettre la fécondation de l'ovule. Ainsi, le volume de liquide séminal est une partie importante de I'analyse du sperme effectuée afin d'évaluer la fertilité mâle. Dans notre article, nous passons en revue l'anatomie et la physiologie de l'éjaculation et les diverses causes (artéfactuelles, structurales et fonctionnelles) d'un faible volume d'éjaculat. Nous présentons ensuite un algorithme complet pour l'évaluation, le diagnostic et le traitement de l'infertilité dans les cas de faible volume de liquide séminal.

\section{Introduction}

Sperm are produced within the testis, in the process of spermatogenesis, then travel through the male reproductive tract (the seminiferous tubules, epididymis, vas deferens, prostate and urethra). As the sperm travel, seminal fluid is added from the seminal vesicles, prostate, testis and epididymis and peri-urethral glands. ${ }^{1}$ The function of the ejaculate is to transport sperm into the female genital tract and to provide a suitable environment for sperm survival during this transit.

An adequate volume of ejaculate is required to carry the male gametes into the female reproductive tract, and thus ejaculate volume is an important component of a semen analysis done to investigate male factor infertility. However, this parameter is often overlooked if other semen abnormalities are also present.

The purpose of this review is to describe the physiology and pathophysiology of conditions leading to low volume ejaculate, to develop a rational approach to the evaluation of these patients and to briefly describe treatment options based on diagnostic category.

\section{Physiology and anatomy of ejaculation}

The ejaculate is composed of secretions from various sources. Two-thirds of the ejaculate volume is contributed by the seminal vesicles, one-third is contributed by the prostate, up to $10 \%$ is derived from the testicle and epididymis, and a small component is derived from the bulbo-urethral glands. ${ }^{2}$

The vas and ducts from the seminal vesicles coalesce to form the paired ejaculatory ducts, which enter the prostatic urethra lateral to the verumontanum. The internal sphincter (bladder neck) is proximal to the ejaculatory duct orifice, and contracts during emission and ejaculation to prevent retrograde ejaculation. The striated sphincter is located distal to the ejaculatory ducts, and opens during ejaculation. $^{2}$ The effector nerves for emission and bladder neck closure are derived from the sympathetic fibres from T10$\mathrm{L} 2$, travel through the hypogastic plexus, and become the peripheral pelvic nerves. Sensory input from the dorsal nerves of the penis travel to the sacral spinal cord (S2-4), and parasympathetic efferents travel through the pudendal nerve (S2-4) to innervate the pelvic floor and periurethral muscle. ${ }^{3}$

The normal ejaculatory process occurs in several steps. During sexual stimulation, there is a progressive rise in external sphincter tone. ${ }^{4}$ Following this, there is a rise in internal spincter tone, and a drop in external spincter tone. Deposition of ejaculate into the posterior urethra (seminal emission) occurs simultaneously. It is also during emission that sperm is rapidly transported from the cauda epididymis and deposited in the posterior urethra. ${ }^{5}$ With the internal spincter closed tightly, a series of involuntary rhythmic contractions of the periurethal muscles occurs, expelling the ejaculate. The closed bladder neck prevents retrograde ejaculation. ${ }^{6}$ As will be discussed, any interruption of the structural 
or functional aspects of this process can affect the ejaculatory volume.

\section{Epidemiology and etiology of low semen volume}

The prevalence of low-volume ejaculate in the population as a whole (fertile or infertile) is unknown. Similarly, the relative frequency of each cause of low-volume ejaculate has not been well defined in the literature. A better understanding of the epidemiology of low-volume ejaculation would be helpful in guiding the workup of these patients, and further studies on this issue are warranted.

In general, the finding of a low-volume ejaculate on semen analysis can be due to artifact, psychogenic causes of ejaculatory dysfunction and various pathologic causes (Table 1).

\section{Artifactual causes of low semen volume}

The timing of collection, as well as the method of collection, can significantly affect the volume of ejaculate on semen analysis.

\section{Timing of collection}

The World Health Organization recommends that the collection be performed after 2 to 3 days of abstinence. ${ }^{7}$ The abstinence interval is very important because in the first 4 days following ejaculation, semen volume increases by $11.9 \%$ per day. ${ }^{8}$

\section{Method of collection}

The collection method is also a determinant of ejaculate volume. The standard collection method is through masturbation into a sterile collection container. The patient

Table 1: Etiology of low semen volume

\begin{tabular}{ll}
\hline Etiology & Features \\
\hline Artifact & Short abstinence period \\
& Incomplete collection
\end{tabular}

\begin{tabular}{ll}
\hline Psychogenic & Anorgasmia \\
\hline Pathologic & Retrograde ejaculation \\
& Structural (damage to bladder neck) \\
& Functional (nerve and neurotransmitter ) \\
Failure of emission (nerve) & Ejaculatory duct obstruction \\
& Congenital ( \pm seminal vesicles or vas anomalies) \\
& Acquired \\
Agenesis or aplasia of the seminal vesicles, \\
prostate \\
Seminal vesicle disease (infection, cysts, ADPKD) \\
Hypogonadism
\end{tabular}

ADPKD $=$ autosomal dominant polycystic kidney disease. may fail to capture all of the ejaculate in the container during collection, and this will lead to a low-volume collection. Patients who are unable or unwilling to collect their sample through masturbation may use intercourse with either coitus interruptus or use of a specifically-designed condom (silastic seminal collection device). It has been shown that ejaculate volume may be higher when collection is done with intercourse and a silastic seminal-collection device than with masturbation or coitus interruptus. ${ }^{9-11}$ The site (home or clinic) at which the sample is collected seems to have no impact on semen volume. ${ }^{12}$

Ensuring an adequate collection of the entire ejaculate is an essential component of the assessment of the patient with a low-volume ejaculate. If there is any doubt, a repeat collection should be obtained. While collection via masturbation is the accepted standard method, if there is concern that the collection via masturbation is not representative of the true ejaculate volume, then the use of a silastic seminal collection device may be helpful.

\section{Psychogenic causes}

Patients with psychogenic anorgasmia may also present with complaints of low-volume or absent ejaculate. ${ }^{13}$ These patients are often subjected to multiple investigations to rule out pathologic causes of a low-volume ejaculate. However, a careful history can help to identify these patients and direct them toward appropriate counselling to address their anorgasmia, without the need for extensive investigations.

\section{Pathologic causes of low semen volume}

\section{Retrograde ejaculation}

Retrograde ejaculation refers to the partial or complete flow of ejaculate into the bladder, rather than an antegrade ejaculation where semen is propelled out the urethra. This can occur due to either structural or functional disruption of the ejaculation process. Retrograde ejaculation can be partial, with some antegrade component, or complete (see below).

Structural changes at the bladder neck are most often due to bladder neck surgery (transurethral resection/incision, bladder neck plasty) or trauma. When the internal sphincter is damaged through one of these processes, it is unable to close during emission and ejaculation, and a retrograde flow of semen (partial or complete) can occur. ${ }^{14}$

Functional changes causing retrograde ejaculation are often due to abnormalities in normal bladder neck closure during ejaculation, which depends on normal functioning of the nerves and neuro-receptors to and within the bladder neck. The function of the bladder neck during ejaculation can be affected by processes altering the nerve input to the sphincter, as well as altering the neuroreceptors within the 
bladder neck. The function of these nerves could be damaged or altered by:

- surgery or trauma (such as pelvic surgery, retroperitoneal lymph node dissection with damage to the sympathetic nerves and spinal cord injury or surgery ${ }^{15}$ ),

- peripheral neuropathy (diabetes is a common cause of peripheral neuropathy, which often leads to progressively more severe retrograde ejaculation and eventually anejaculation and failure of emission ${ }^{16}$ ),

- drugs (notably alpha blockers, ${ }^{17}$ which can act to prevent muscle contraction at the bladder neck and lead to retrograde ejaculation; this is usually reversible with cessation of the medication).

\section{Anejaculation}

Complete absence of ejaculation can be due to complete retrograde ejaculation, failure of emission or anorgasmia.

Complete retrograde ejaculation may be due to any of the causes listed above. Failure of emission is usually due to a neurologic cause, with an interruption of the sympathetic nerves or output. This may be the result of neurological damage at the spinal cord level (spinal cord injury (SCI), spinal dysraphism, demyelinating disease, tumours and degenerative conditions), at the level of post-ganglionic sympathetic fibres (e.g., sympathetic nerve damage with retroperitoneal surgery or trauma, notably retroperitoneal lymph node dissection).

While most of these patients have a clear history of an event (trauma, surgery) that preceded their ejaculatory problem, some patients may have an occult cause for anejaculation, such as spina bifida occulta/tethered cord syndrome.

Finally, anorgasmia will result in absence of ejaculation. While this is primarily a psychogenic condition, other etiologies include use of selective serotonin reuptake inhibitors ${ }^{18}$ and neurologic disease or injury. ${ }^{19}$

\section{Ejaculatory duct obstruction}

Classically, ejaculatory duct obstruction (EDO) refers to complete obstruction of both ejaculatory ducts, leading to a low-volume, azoospemic ejaculate. More subtle forms, such as unilateral complete obstruction, bilateral partial obstruction and functional obstruction may also cause lowvolume ejaculate, but the ejaculate often contains sperm. Causes can be divided into congenital and acquired, and include atresia of the ducts, Mullerian and Wolffian duct cycts, seminal vesicle calculi, postsurgical or postinflammatory scarring, or calcification near the verumontanum.

\section{Congenital absence of the vas/seminal vesicles}

Congenital bilateral absence of the vas deferens (CBAVD) is typically found in association with absence or hypoplasia of the seminal vesicles. ${ }^{20}$ Since the majority of ejaculate volume is derived from the seminal vesicles, absence or hypoplasia of these structures can lead to a low volume of ejaculate. Typically, men with the absence of the vas deferens have low-volume (often less than $0.5 \mathrm{~mL}$ ), acidic semen. These men may also have renal anomalies. Up to $80 \%$ of men with CBAVD carry at least one genetic mutation of the cystic fibrosis (CF) gene. ${ }^{20}$

Congenital unilateral absence of the vas deferens (CUAVD) may also present with low semen volume and absence or hypoplasia of the ipsilateral seminal vesicles. ${ }^{21}$ It may also be associated with cystic fibrosis gene mutations, and may be associated with other Wolffian duct abnormalities, such as abnormalities or absence of the ipsilateral kidney and ureter.

\section{Hypogonadism}

The Wolffian structures (epididymis, vas deferens, seminal vesicles, ejaculatory ducts) and the prostate are dependent on testosterone and dihydrotestosterone, respectively, for development and maintenance of function. Conditions leading to low levels of testosterone (primary or secondary hypogonadism) can lead to decreased secretions from these organs and, consequently, low-volume ejaculate. ${ }^{22,23}$

\section{Idiopathic}

At times, the cause of a low-volume ejaculate cannot be identified. However, a careful history and physical exam, combined with some basic investigations, can often help to identify an underlying cause.

\section{Patient presentation and evaluation}

The finding of a low-volume ejaculate is most commonly made in the process of a fertility evaluation, where patients have performed one or more semen analyses. Less commonly, a patient may present with low-volume or absent ejaculate as a primary complaint. While the clinical assessment of male fertility is somewhat extensive, we will focus specifically on the evaluation of the ejaculate volume.

The approach to the patient with a finding of low-volume ejaculate follows from the etiologic categories listed above. The history should serve to rule out potential psychogenic and artifactual causes of low-volume ejaculate, and then identify associated symptoms that may lead to one of the specific diagnoses (Box 1). The history will help to determine if the low semen volume is likely an artifact, psychogenic or truly pathologic. 


\section{Box 1. The patient's history}

Is this an artifact?

Question the patient about the abstinence period, collection methods used (masturbation, SCD, etc) and completeness of collection. Asking whether the patient feels that the volume of ejaculate produced during specimen collection is similar to the volume produced during normal sexual activity may help to identify patients whose semen volume is low only during specimen collection.

Is there a psychogenic cause?

The history should carefully assess overall sexual function (libido, erection, orgasm, ejaculation) to identify potential contributing psychogenic conditions. One important factor to determine is if the patient actually has an orgasm.

Is there a pathologic cause?

The pathologic causes include retrograde ejaculation, failure of emission and alterations in the Wolffian duct structures (seminal vesicles, ejaculatory ducts and vas deferens).

Is there evidence for retrograde ejaculation or failure of emission? A history of cloudy urine following ejaculation is often associated with RE, and this should be elicited. History of surgery, trauma or disease that might affect the sympathetic nerves or the bladder neck should be elicited. A finding of absent ejaculate must prompt questioning regarding spinal or neurologic disease, and previous prostate surgery. Other symptoms of neurologic dysfunction (leg weakness, bladder/bowel dysfunction) should also be sought.

Is there evidence of an EDO or absence of the Wolffian ducts?

A history of prostatic surgery or infection, and symptoms such as pain with ejaculation or hematospermia, can be associated with EDO. While most patients with CBAVD do not have clinical CF, 24 history of respiratory illness/symptoms, as well as a family history of CF and infertility, is useful.

Are there symptoms of hypogonadism?

Low testosterone levels can be associated with various symptoms of hypogonadism, and these should be elicited (low energy, mood changes, weakness, ED, decreased libido).

$\mathrm{SCD}=$ silastic seminal collection device; $\mathrm{RE}=$ retrograde ejaculation; $\mathrm{EDO}=$ ejaculatory duct obstruction; CBAVD = Congenital unilateral absence of the vas deferens; CBAVD = congenital bilateral absence of the vas deferens; $C F=$ cystic fibrosis; $E D=$ erectile dysfunction.

\section{Physical examination}

Physical examination should include an assessment of androgenization status, external genital exam including palpation of the vas, digital rectal examination, and focused lowerextremity neurologic exam. Physical examination is often normal, but important notable findings can include inguinal/scrotal scars, small testes volume, testicular mass and varicocele(s). In the patient presenting with lowvolume azoospermia, palpable prostatic abnormalities (midline cyst) and absence of the vas deferens (unilateral or bilateral) may be identified.

\section{Semen testing}

Assessment of other parameters on the semen analysis can also be helpful in identifying potential underlying causes. A finding of normal semen parameters can be associated with collection problems or partial retrograde ejaculation.
Oligo/astheno/teratospermia can be seen with partial ejaculatory duct obstruction or hypogonadism. Azoospermia is suggestive of ejaculatory duct obstruction, absence of the vas deferens, failure of emission and complete retrograde ejaculation. Absence of fructose and an acidic $\mathrm{pH}$ are suggestive of ejaculatory duct obstruction or seminal vesicle pathology. ${ }^{25}$ Of course, there are other conditions associated with abnormalities on semen analysis (e.g., varicocele, epididymal obstruction, testes failure), so none of these findings are specific causes of low ejaculate volume.

\section{Evaluation}

The first step in evaluating a patient with low-volume ejaculate is to ensure that two properly collected semen analyses have been performed. If there is a large discrepancy between the results, a third should be performed.

Post-ejaculate urinalysis (PEU) can be done to identify patients with potential retrograde ejaculation. The patient is asked to empty his bladder, and then a standard semen collection is performed. The patient then voids into a collection container, which is then analyzed for sperm. Typically, the urine is centrifuged at $300 \mathrm{~g}$ for 10 minutes, and then the pellet is re-suspended in $1 \mathrm{~mL}$ of saline. A sperm concentration and total sperm count can then be determined.

While the PEU is easily performed and noninvasive, the interpretation of its results remains controversial. Sperm in the urine can be due to retrograde ejaculation, or due to the expulsion of urethral sperm during voiding. ${ }^{26}$ Various cut-offs for PEU sperm count have been used to define retrograde ejaculation, but there is no clear consensus and very little data on the subject. One method is to establish a threshold urine sperm count above which retrograde ejaculation is diagnosed. The other is to define a cut-off for the percentage of sperm in the urine, relative to the total sperm count of the urine and the ejaculate. However, it has been shown that $65 \%$ of fertile men ${ }^{27}$ and $73 \%$ to greater than $90 \%$ of infertile men ${ }^{27,28}$ have some sperm on PEU. Sigman and colleagues compared urine and ejaculate sperm counts in fertile and infertile men. ${ }^{27}$ They found no difference between the absolute sperm count in the urine, and both groups had the same percentage of sperm in the urine (15\%). Thus, except in the rare case of a man who is azoospermic but has sperm in the urine, the diagnosis of retrograde ejaculation based on the PEU remains difficult.

Transrectal ultrasound (TRUS) gives excellent images of the prostate, seminal vesicles and vas deferens. This test is most useful when ejaculatory duct obstruction is suspected, usually in the patient with low-volume azoospermia. ${ }^{29}$ As well, it can be used to determine associated seminal vesicle anatomy in the patient with CUAVD or CBAVD. Findings suggestive of ejaculatory duct obstruction include dilated 
seminal vesicles (diameter greater than $1.5 \mathrm{~cm}$ ) and ejaculatory ducts (diameter greater than 2 to $3 \mathrm{~mm}$ ), especially when associated with a cyst or calcification along the course of the duct. ${ }^{29}$ Unfortunately, dilated seminal vesicles and ejaculatory ducts are not seen in all cases of ejaculatory duct obstruction, and these structures can be dilated in the absence of obstruction in up to $50 \%$ of cases. ${ }^{30}$ Furthermore, partial or unilateral ejaculatory duct obstruction (which are controversial in their own right) can give similarly confusing appearances on TRUS. ${ }^{31}$ However, due to its relatively low cost and availability, TRUS is often the first test done in patients with low-volume ejaculate and azoospermia to rule out ejaculatory duct obstruction. Its role in patients with lowvolume ejaculate without azoospermia is not well defined.
Additional tests that can be done in the case where TRUS results are inconclusive include magnetic resonance imaging, $^{32}$ seminal vesicle aspiration ${ }^{31}$ and seminal vesiculography. ${ }^{33}$ These is no consensus as to the value of these tests, and as such they are not recommended as routine investigations.

In patients with a suspected occult neurologic lesion (e.g., tethered cord), a neurology consult should be obtained and spinal imaging, such as $\mathrm{x}$-rays and magnetic resonance imaging, can be obtained.

In patients with CUAVD or CBAVD as determined by physical examination, cystic fibrosis genetic analysis should be done. An abdominal ultrasound can identify patients with renal anomalies (especially with CUAVD).

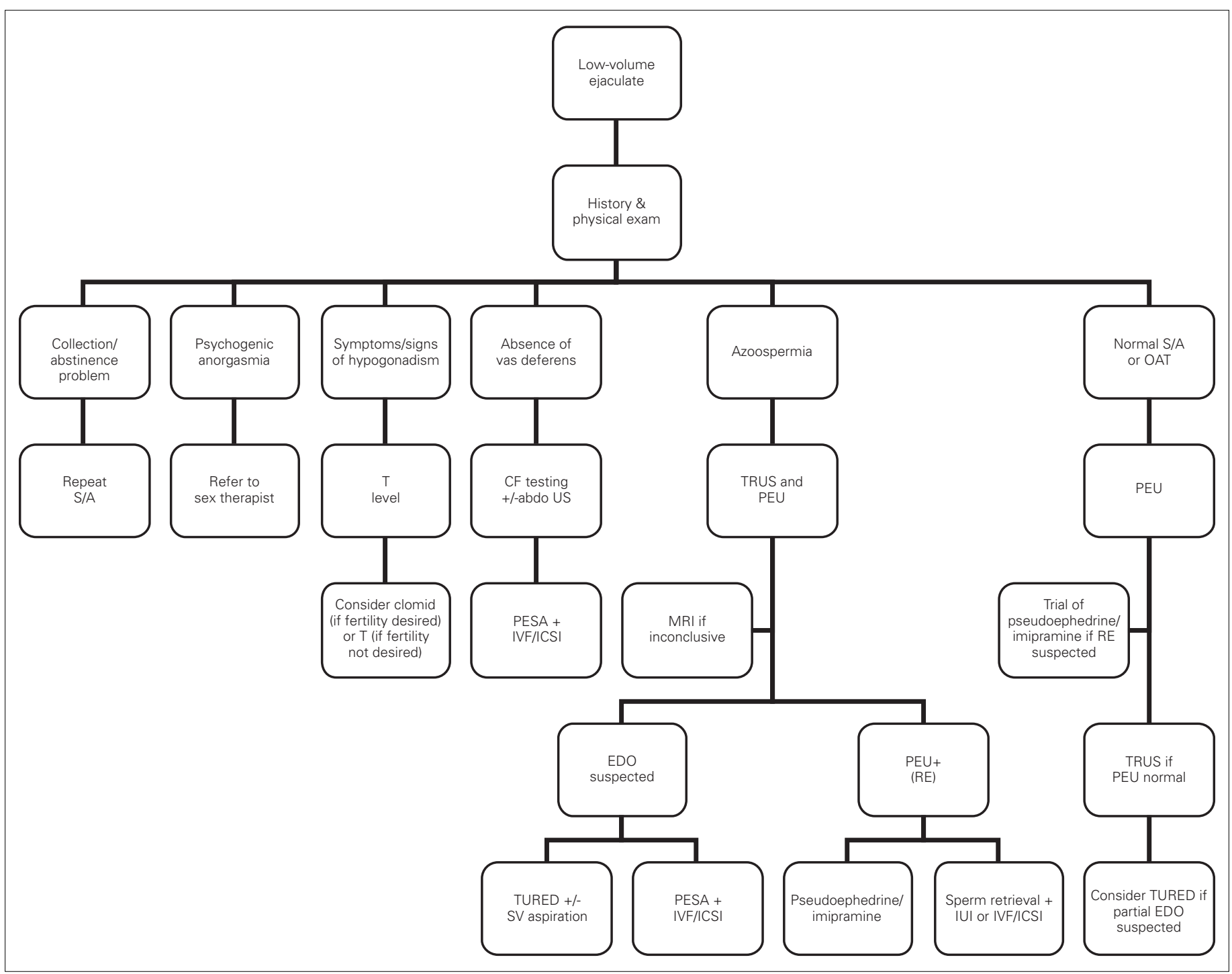

Fig. 1. Algorithm for the evaluation and treatment of a man presenting with low-volume ejaculation. $S / A=$ semen analysis; $0 A T=$ oligoasthenoteratozoospermia; Abdo US = abdominal ultrasound; $C F=$ cystic fibrosis; $P E U$ = post-ejaculate urinalysis; TRUS = transrectal ultrasound; PESA = percutaneous epididymal sperm aspiration; IVF = in vitro fertilization; ICSI = intracytoplasmic sperm injection; $\mathrm{MRI}=$ magnetic resonance imaging; RE = retrograde ejaculation; TURED = transurethral resection of the ejaculatory ducts; $S V$ = seminal vesicle; IUI = intrauterine insemination; EDO = ejaculatory duct obstruction. 
An algorithm for the investigation of patients presenting with low volume ejaculate is presented in Figure 1. A logical, stepwise approach is preferable to a "shot-gun" approach to minimize the number of investigations performed and to minimize patient discomfort and inconvenience.

\section{Treatment}

A full discussion of the treatment options for every cause of low-volume ejaculate is beyond the scope of this review article; however, we briefly discuss the management options for each of the conditions discussed above.

Anorgasmia in the absence of any identifiable pathology, such as hypogonadism, is usually due to psychologic causes. A referral to a sex therapist may be helpful for these men. ${ }^{13}$

Retrograde ejaculation from functional causes is often amenable to oral pharmacotherapy. The two agents most commonly used are pseudoephedine and imipramine; they can be used individually or in combination. ${ }^{34-36}$ These agents have sympathomimetic properties and increase the tone at the bladder neck, and can also stimulate seminal emission. Imipramine can be taken (25 mg orally daily, escalated to $50 \mathrm{mg}$ orally daily if no response) for 7 days prior to attempted ejaculation, and pseudoephedrine (30 mg orally) may be taken daily or 30 to 60 minutes prior to ejaculation. ${ }^{2}$ Re-establishing antegrade ejaculation in patients with complete retrograde ejaculation with either pseudoephedrine or imipramine was successful in $47.8 \%$ and $38.5 \%$, respectively, irrespective of the etiology of retrograde ejaculation. ${ }^{35}$ Retrograde ejaculation from structural causes is often not amenable to pharmacologic treatment. For refractory retrograde ejaculation, sperm retrieval from the urine for use with intrauterine insemination (IUI) or in vitro fertilization (IVF) may be required. ${ }^{37}$ Since the acidic urinary $\mathrm{pH}$ is hostile to sperm, alkalinization is required prior to specimen collection. The patient should take oral sodium bicarbonate, $500 \mathrm{mg}, 12$ and 2 hours prior to donation. Alternatively, bladder catheterization with the instillation of sperm-friendly buffer solution can be done. ${ }^{38}$

Anejaculation, as in retrograde ejaculation, may also respond to oral treatment, especially in patients with diabetes mellitus who have progressed from retrograde ejaculation to anejaculation. ${ }^{36}$ Other alternatives in these patients are penile vibratory stimulation or electroejaculation, especially in patients with spinal cord injury. ${ }^{39,40}$ Sperm retrieval with either percutaneous epididymal sperm aspiration (PESA) or testicular sperm extraction (TESE) and subsequent IVF is also possible. ${ }^{41,42}$ One analysis of the cost-effectiveness of electroejaculation plus intra-uterine insemination versus surgical sperm retrieval plus IVF showed that electroejaculation plus IUI was superior if anesthesia was not required, but that sperm retrieval plus IVF was superior if anesthesia was required for electroejaculation. ${ }^{43}$
Ejaculatory duct obstruction can be treated with transurethral resection of the ejaculatory ducts. ${ }^{44}$ This treatment is highly effective in patients with ejaculatory duct obstruction demonstrated on transrectal ultrasonography who have low-volume azoospermia, ${ }^{45}$ although there are small risks of hematuria, incontinence, rectal injury and watery ejaculate. ${ }^{45,46}$ Retrograde ejaculation can also occur, but sperm can be obtained from the urine and used for IUI or IVF. In patients with suspected partial ejaculatory duct obstruction (low-volume ejaculate but not azoospermia), there is a risk of converting the patient to complete azoospermia, likely from scarring of the ejaculatory duct. It should be noted that some patients (10\% to $15 \%$ ) with ejaculatory duct obstruction may have concurrent epididymal obstruction and will not respond to transurethral resection of the ejaculatory ducts (TURED) alone. ${ }^{30}$ Overall, semen parameters in patients with partial or complete ejaculatory duct obstruction have been shown to improve in 59\% to $94 \%$ of cases, ${ }^{45,46}$ with pregnancy rates ranging from $20 \%$ to $30 \%{ }^{7}$ If TURED is unsuccessful or is declined by the patients, sperm retrieval with PESA and subsequent IVF is a viable alternative. Recently, seminal vesicle aspiration with sperm retrieval for IVF has been described, but remains experimental. ${ }^{47}$

Seminal vesicle/vas deferens malformations (hypoplasia, aplasia, cystic fibrosis) are generally not amenable to surgical reconstruction. In patients with azoospermia, sperm retrieval with PESA or TESE and IVF- intracytoplasmic sperm injection often are required. ${ }^{48}$

Hypogonadism is typically treated with testosterone administration. However, testosterone is contraindicated in men seeking fertility since it suppresses the hypothalamicpituitary-gonadal axis and often leads to azoospermia. In hypogonadal men with low-volume ejaculate, treatment with clomiphene citrate may increase testosterone levels, but its effects on ejaculate volume are unknown, although one report found no difference in ejaculate volume before and after treatment. ${ }^{49}$

\section{Conclusion}

Ejaculate volume is an important component of the semen analysis and evaluation of the infertile man, but is often overlooked if other abnormalities are also present on semen analysis (e.g., low sperm count). A careful history and physical examination can help identify most causes, and can help guide subsequent investigations. Treatment to correct the problem may be possible in some patients and, in others, identification of important medical conditions (e.g., cystic fibrosis mutations, spinal cord disease) may occur.

From the *Male Reproductive Medicine and Surgery; ${ }^{\dagger}$ Chief of Urology, Mount Sinai Hospital, Professor, Division of Urology, University of Toronto, Toronto, ON 
Competing interests: None declared.

This paper has been peer-reviewed.

\section{References}

1. Sigman M, Jarow JP. Male Infertility. In: A. Wein, ed. Campbell-Walsh Urology. Philadelphia: Saunders; 2006:chap. 19

2. Ohl DA, Quallich SA, Sønksen J, et al. Anejaculation and retrograde ejaculation. Urol Clin North Am 2008;35:211-20, viii.

3. Thomas AJ Jr, Ejaculatory dysfunction. Fertil Steril 1983;39:445-54.

4. Sonksen J, Ohl DA, Wedemeyer G. Sphincteric events during penile vibratory ejaculation and electroejaculation in men with spinal cord injuries. J Urol 2001;165:426-9.

5. Bruschini H, Schmidt RA, Tanagho EA. Studies on the neurophysiology of the vas deferens. Invest Urol 1977;15:112-6.

6. Böhlen D, Hugonnet $\mathrm{CL}$, Mills RD, et al. Five meters of $\mathrm{H}(2) 0$ : the pressure at the urinary bladder neck during human ejaculation. Prostate 2000;44:339-41.

7. Pryor JP, Hendry WF. Ejaculatory duct obstruction in subfertile males: analysis of 87 patients. Fertil Steril 1991;56:725-30.

8. Carlsen E, Petersen JH, Andersson AM, et al. Effects of ejaculatory frequency and season on variations in semen quality. Fertil Steril 2004;82:358-66.

9. Gerris J. Methods of semen collection not based on masturbation or surgical sperm retrieval. Hum Reprod Update 1999:5:211-5.

10. Zavos PM, Kofinas GD, Sofikitis NV, et al. Differences in seminal parameters in specimens collected via intercourse and incomplete intercourse (coitus interruptus). Fertil Steril 1994;61:1174-6.

11. Sofikitis NV, Miyagawa I. Endocrinological, biophysical, and biochemical parameters of semen collected via masturbation versus sexual intercourse. J Androl 1993;14:366-73.

12. Elzanaty $\mathrm{S}$, Malm J. Comparison of semen parameters in samples collected by masturbation at a clinic and at home. Fertil Steril 2008:89:1718-22.

13. McMahon CG, Abdo C, Incrocci L, et al. Disorders of orgasm and ejaculation in men. J Sex Med 2004; 1:58-65.

14. Miner $M$, Rosenberg MT, Perelman MA. Treatment of lower urinary tract symptoms in benign prostatic hyperplasia and its impact on sexual function. Clin Ther 2006;28:13-25.

15. Terrone C, Castelli E, Aveta P, et al. Iatrogenic ejaculation disorders and their prevention. Minerva Urol Nefrol 2001;53:19-28.

16. Sexton WJ, Jarow JP. Effect of diabetes mellitus upon male reproductive function. Urology 1997;49: 508-13.

17. Giuliano F. Impact of medical treatments for benign prostatic hyperplasia on sexual function. BJU Int 2006;97(Supp|2):34-8; discussion 44-5.

18. Stadler T, Bader M, Uckert $S$, et al. Adverse effects of drug therapies on male and female sexual function. World J Urol 2006;24:623-9.

19. Wolters JP, Hellstrom WJ. Current concepts in ejaculatory dysfunction. Rev Urol 2006;8 (Suppl4): S18-25.

20. Wilschanski $M$, Corey $M$, Durie $P$, et al. Diversity of reproductive tract abnormalities in men with cystic fibrosis. JAMA 1996;276:607-8.

21. Kolettis PN, Sandlow Jl. Clinical and genetic features of patients with congenital unilateral absence of the vas deferens. Urology 2002;60:1073-6.

22. Tash JA, McGovern JH, Schlegel PN. Acquired hypogonadotropic hypogonadism presenting as decreased seminal volume. Urology 2000;56:669.

23. Tulandi T, McInnes RA. Induction of fertility in a man with hypogonadotropic hypogonadism with very low seminal volume. Fertil Steril 1986;46:730-3.

24. Daudin M, Bieth E, Bujan L, et al. Congenital bilateral absence of the vas deferens: clinical characteristics, biological parameters, cystic fibrosis transmembrane conductance regulator gene mutations, and implications for genetic counseling. Fertil Steril 2000;74:1164-74.
25. Ozgök Y, Tan MO, Kilciler $M$, et al. Diagnosis and treatment of ejaculatory duct obstruction in male infertility. Eur Urol 2001;39:24-9.

26. Ariagno II, Mendeluk GR, Pugliese MN, et al. The only presence of sperm in urine does not imply retrograde ejaculation. Arch Androl 2005;51:431-6.

27. Sigman $M$, Boyle K, Jarow JP. Prevalence of sperm in the post-ejaculatory urine of fertile and subfertile men. Urology 2008;71:110-2.

28. Weissenberg $R$, Rozenman $H$, Hova $M$, et al. The diagnostic value of sperm in post-ejaculatory urine. Int J Androl 1984;7:142-8.

29. Schroeder-Printzen I, Ludwig $M$, Köhn $F$, et al. Surgical therapy in infertile men with ejaculatory duct obstruction: technique and outcome of a standardized surgical approach. Hum Reprod 2000;15: 1364-8.

30. Smith JF, Walsh TJ, Turek PJ. Ejaculatory duct obstruction. Urol Clin North Am 2008;35:221-7, viii.

31. Engin $G$, Celtik $M$, Sanli 0 , et al. Comparison of transrectal ultrasonography and transrectal ultrasonography-guided seminal vesicle aspiration in the diagnosis of the ejaculatory duct obstruction. Fertil Steril 2009:92:964-70.

32. Engin G, Kadio lu A, Orhan I, et al. Transrectal US and endorectal MR imaging in partial and complete obstruction of the seminal duct system. A comparative study. Acta Radiol 2000;41:288-95.

33. Purohit RS, Wu DS, Shinohara K, et al. A prospective comparison of 3 diagnostic methods to evaluate ejaculatory duct obstruction. J Urol 2004;171:232-5; discussion 235-6.

34. Ochsenkuhn R, Kamischke A, Nieschlag E. Imipramine for successful treatment of retrograde ejaculation caused by retroperitoneal surgery. Int J Androl 1999;22:173-7.

35. Arafa $\mathrm{M}$, El Tabie 0 . Medical treatment of retrograde ejaculation in diabetic patients: a hope for spontaneous pregnancy. J Sex Med 2008;5:194-8.

36. Kamischke A, Nieschlag E. Update on medical treatment of ejaculatory disorders. Int J Androl 2002;25: 333-44.

37. Aust TR, Brookes $S$, Troup $S A$, et al. Development and in vitro testing of a new method of urine preparation for retrograde ejaculation; the Liverpool solution. Fertil Steril 2008;89:885-91.

38. Suminen JJ, Kilkku PP, Taina EJ, et al. Successful treatment of infertility due to retrograde ejaculation by instillation of serum-containing medium into the bladder. A case report. Int J Androl 1991;14: $87-90$.

39. Löchner-Ernst D, Mandalka B, Kramer G, et al. Conservative and surgical semen retrieval in patients with spinal cord injury. Spinal Cord 1997;35:463-8.

40. Chung PH, Palermo G, Schlegel PN, et al. The use of intracytoplasmic sperm injection with electroejaculates from anejaculatory men. Hum Reprod 1998;13:1854-8.

41. Kanto $S$, Uto $H$, Toya $M$, et al. Fresh testicular sperm retrieved from men with spinal cord injury retains equal fecundity to that from men with obstructive azoospermia via intracytoplasmic sperm injection. Fertil Steril 2009;92:1333-6.

42. Kafetsoulis A, Brackett NL, Ibrahim E, et al. Current trends in the treatment of infertility in men with spinal cord injury. Fertil Steril 2006;86:781-9.

43. Ohl DA, Wolf $\mathrm{L}$, Menge AC, et al. Electroejaculation and assisted reproductive technologies in the treatment of anejaculatory infertility. Fertil Steril 2001;76:1249-55.

44. Turek P. Seminal vesicle and ejaculatory duct surgery. In: Graham S, ed. Glenn's Urologic Surgery. Philadelphia: Lippincott, Williams \& Wilkins; 2006:439-45.

45. Turek PJ, Magana J0, Lipshultz LI. Semen parameters before and after transurethral surgery for ejaculatory duct obstruction. J Urol 1996;155:1291-3.

46. Kadioglu A, Cayan S, Tefekli A, et al. Does response to treatment of ejaculatory duct obstruction in infertile men vary with pathology? Fertil Steril 2001;76:138-42.

47. Boehlen D, Schmid HP. Novel use of fine needle aspiration of seminal vesicles for sperm retrieval in infertile men. Urology 2005;66:880.

48. Hubert D, Patrat C, Guibert J, et al. Results of assisted reproductive technique in men with cystic fibrosis. Hum Reprod 2006;21:1232-6.

49. Breznik R, Borko E. Effectiveness of antiestrogens in infertile men. Arch Androl 1993;31:43-8.

Correspondence: Dr. Keith Jarvi, Chief of Urology, Mount Sinai Hospital, Professor, Division of Urology, University of Toronto, Murray Koffler Urologic Wellness Centre, 60 Murray Street, 6th Floor, Box 19, Toronto, 0N M5T 3L9; fax: 416-586-4776; kjarvi@mtsinai.on.ca 